\title{
Novel antibiotics: Are we still in the pre-post-antibiotic era?
}

\author{
R. Draenert $\cdot$ U. Seybold $\cdot$ E. Grützner $\cdot$ J. R. Bogner
}

Received: 23 December 2014 / Accepted: 11 February 2015 / Published online: 21 February 2015

(C) The Author(s) 2015. This article is published with open access at Springerlink.com

\begin{abstract}
Purpose Therapeutic efficacy and safety in infections due to multidrug-resistant bacteria can be improved by the clinical development of new compounds and devising new derivatives of already useful antibiotics. Due to a striking global increase in multidrug-resistant Gram-positive but even more Gram-negative organisms, new antibiotics are urgently needed.
\end{abstract}

Methods This paper provides a review of novel antibiotic compounds which are already in clinical development, mainly in phase III clinical trials.

Conclusion Each of these new trials increases the possibility of new antibiotics receiving approval.

Keywords Tedizolid Ceftolozane - Omadacycline ·

Avibactam $\cdot$ Cadazolid

\section{Introduction}

New antibiotics are urgently needed due to the alarming development of resistance against all antibiotics on the market and in clinical use $[1,2]$. While this has been the case since the detection of antibiotics, recently the gap has widened due to the fact that new antibiotic drug classes have barely been introduced among others. Moreover, the prevalence of difficult to treat resistant and multi-resistant

This review is dedicated to Prof. Dr. med. G. Fätkenheuer for his anniversary with our best wishes!

R. Draenert $(\varangle) \cdot$ U. Seybold · E. Grützner · J. R. Bogner Sektion Infektiologie, Medizinische Klinik und Poliklinik IV, Klinikum der Universität München, Pettenkoferstr. 8a, 80336 Munich, Germany

e-mail: rika.draenert@med.uni-muenchen.de nosocomial organisms is rapidly increasing both for Gram-negative and Gram-positive bacteria. However, at the moment, Gram-negative bacilli are the greater threat. While the prevalence of methicillin-resistant Staphylococcus aureus (MRSA) has plateaued in many countries and is even declining in some, the frequency of enterobacteriaceae that exhibit high rates of antibiotic resistance including broad-spectrum beta-lactamases (extended-spectrum beta-lactamase, ESBL) and even carbapenemases is rapidly increasing and poses imminent threat for patients with infections due to these organisms. For this reason, a large number of long-known beta-lactam antibiotics based on penicillins or cephalosporins have become ineffective in many instances, even when combined with conventional inhibitors of beta-lactamases like clavulanic acid or tazobactam [1].

In the past couple of years, pessimism has been spreading with respect to the pipeline of new antimicrobials, and many pharmaceutical companies have declared that they will not continue to develop new antibacterial compounds. At present, the situation has changed positively, and those observing preclinical and clinical development strategies and activities have reason to be more optimistic. Several compounds have been developed in various drug classes and against resistant organisms in the whole spectrum of multidrug-resistant (MDR) bacteria (Fig. 1).

\section{Compounds against Gram-positive bacteria}

Novel long acting lipoglycopeptides oritavancin and dalbavancin

Acute bacterial skin and skin structure infections (ABSSSIs) are among the most frequent indications 


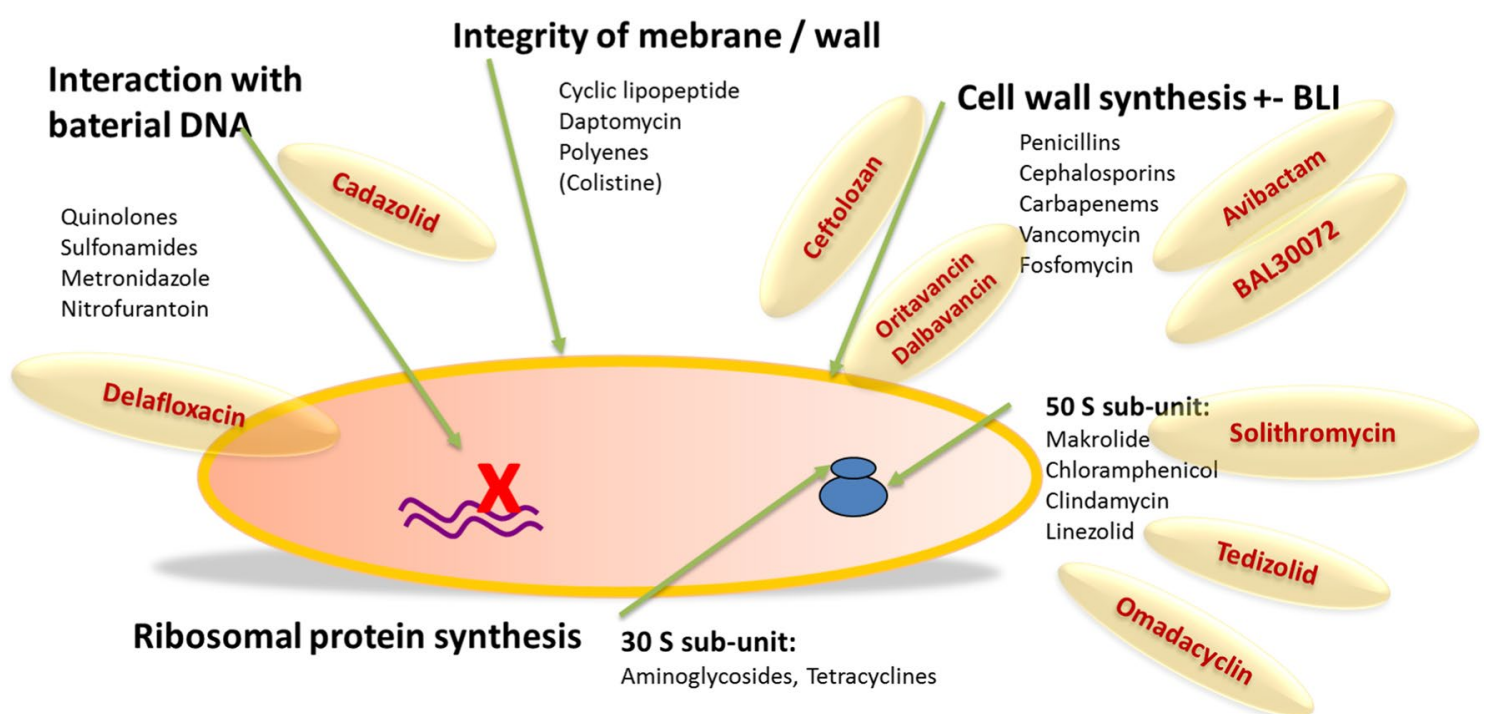

Fig. 1 Schematic antibiotic action: new compounds in an overview

for antimicrobial therapy. The causative agents are usually Gram-positive bacteria including MRSA for which there are a number of treatment options that may be quite demanding with respect to application, dosing frequency, monitoring requirements, and duration of treatment. With the advent of novel lipoglycopeptides (in addition to teicoplanin, which has been available in Europe since 1992 and telavancin, which has received FDA approval in 2009), characterized by a concentration-dependent bactericidal activity and an extended elimination half-life, therapy of ABSSSI may become more easily manageable.

Oritavancin has (at least) three distinct mechanisms of action, namely inhibition of transglycosylation (like vancomycin), inhibition of transpeptidation (like beta-lactams), and disruption of cell membrane integrity (like telavancin) [3]. These result in rapid bactericidal activity against a number of Gram-positive pathogens. It also has a very long terminal half-life of $>300 \mathrm{~h} \mathrm{[4]}$ and demonstrated potent bactericidal activity of a single $1200 \mathrm{mg}$ dose in an in vitro PK/PD model [5]. It is not metabolized, and there is no need for dose adjustment for renal or moderate hepatic impairment. This set of characteristics allows for very easy administration especially in an outpatient setting. Following a phase II study that did not support the daily administration of oritavancin [6], the recent phase III SOLO I trial involving 954 patients in the mITT population demonstrated non-inferiority of a single $1200 \mathrm{mg}$ i.v. dose of oritavancin versus 7-10 days of twice daily i.v. vancomycin for ABSSSI with respect to all three efficacy end points including cure [7]. This held true for a variety of subgroup analyses. While nausea was somewhat more common in the oritavancin group (11 vs. $8.9 \%$ ), there was no statistically significant difference with respect to safety or tolerability in general. Oritavancin therefore has the potential to be used as single-shot treatment for ABSSSIs virtually eliminating adherence issues. As stated for the SOLO I as well as the SOLO II trial, the prolonged half-life of oritavancin was not associated with any safety issues including the 60 day follow-up period [7].

Dalbavancin is another semisynthetic lipoglycopeptide and has been evaluated for skin and soft tissue/skin structure infections $[8,9]$ as well as catheter-associated blood stream infections [10] already since the early 2000s. It has been shown to have only a minor impact on the gut flora [11]. Its terminal half-life of about 2 weeks [12] also allows for extended dosing intervals. Recently, the twin-phase III DISCOVER-1 and DISCOVER-2 trials for ABSSSI with 1312 patients in the pooled analysis were published. They demonstrated non-inferiority of two single doses of dalbavancin given 1 week apart compared to a standard twicedaily treatment regimen of i.v. vancomycin followed by an optional switch to p.o. linezolid for a total of 10-14 days [13]. This was true for both the primary end point of early clinical success and end of treatment success, independent of causative pathogen or comorbidity. Non-inferiority of a two-dose regimen is even more remarkable when considering that about half the patients met the criteria for systemic inflammatory response syndrome (SIRS). Dalbavancin had a favorable safety profile with both fewer adverse events and fewer patients experiencing adverse events. Nausea was noted to be the most frequent adverse event in the dalbavancin group occurring in $2.5 \%$ of patients. Notably, the use of dalbavancin was associated with a significantly lower mortality ( 0.2 vs. $1.1 \%)$. This has led to the FDA approval of dalbavancin for ABSSSI caused by $S$. aureus and S. pyogenes in May 2014. 
Tedizolid

Tedizolid phosphate is a new oxazolidinone compound. Its mechanism of action is similar to that of linezolid, which was the first drug in this class: It acts by inhibition of the ribosomal protein synthesis of bacteria. The exact mechanism of blockade is located at the 50S subunit of the bacterial ribosome, which is directly targeted by oxazolidinones in a way that inhibits binding of the tRNA by a conformational change in the binding moiety. Thereby, the protein synthesis chain is terminated $[14,15]$. The active compound of tedizolid emerges by cleavage of the phosphate moiety of the prodrug. Tedizolid is active against Grampositive bacteria, including MRSA. Vancomycin-resistant organisms and even linezolid-resistant MRSA have been found to be susceptible to the new drug [15-17].

Substantial pharmacological data on tedizolid show its suitability for clinical use as an antibiotic. Pooled analyses of intravenous and oral application have shown correlations of drug levels with clinical success. The absolute bioavailability of tedizolid is higher than $80 \%$ which is comparable to linezolid [14, 17-20]. More positive pharmacological data show a lower interaction potential of tedizolid as compared to linezolid, e.g., no interaction with inhibitors of the monoamino-oxidase (MAO-inhibitors). The long halflife of tedizolid in combination with a high bioavailability offers the realistic rationale for once daily administration. With a 10- to 12-h half-life, drug levels of tedizolid above the MIC can still be accomplished at the end of a 24-h dose interval. About $80 \%$ of the drug is eliminated via the gut and about $20 \%$ renally.

Because the compound has good tissue penetration, one clinical area of use is ABSSSI.

The two-phase III ESTABLISH 1 and ESTABLISH 2 trials showed non-inferiority compared to linezolid, leading to approval of the new drug by the FDA in June 2014 [15, 16]. In ESTABLISH 2, a total of 666 patients with ABSSSI were recruited in nine countries with 58 participating treatment sites. A 1:1 randomization allocated patients in a double-blind manner to either tedizolid $200 \mathrm{mg}$ intravenously followed by oral medication over 6 days or to linezolid at a dose of $600 \mathrm{mg}$ by mouth twice daily over a period of 10 days. Stepdown from intravenous to oral medication could take place if the criteria of early clinical response were fulfilled. Early clinical response was defined as a reduction in the inflamed area by at least $20 \%$ in the first 3 days. This criterion was reached roughly with equal frequency in both trial arms: In the tedizolid arm $85 \%$ and in the linezolid arm $83 \%$ of all patients had early clinical response. Regarding adverse events and safety issues, there was a significantly lower incidence of gastrointestinal adverse reactions in the tedizolid group (16 vs. $23 \%$ ) [16, $17,21]$.
In addition to clinical efficacy data, there have been studies investigating pharmacological issues in special populations: Patients with renal failure and with hepatic insufficiency have been subject to pharmacokinetic sampling. The results show that no dose adjustment is necessary in patients with chronic renal failure with and without hemodialysis treatment. Only $10 \%$ of the antibiotics were removed by dialysis. In patients with hepatic impairment, a moderate increase in drug levels in the range of 22-34\% was observed [19].

\section{Drugs against Gram-negative bacteria and broad-spectrum antibiotics}

\section{BAL30072}

BAL30072 is a monosulfactam antibiotic, which exhibits activity against carbapenem-resistant Enterobacteriaceae and non-fermenters [22-24]. The chemical structure of BAL30072 is related to that of aztreonam. BAL 30072 belongs to the group of beta-lactam antibiotic and inhibits bacterial cell wall synthesis. In vitro tests of combinations with beta-lactamase inhibitors such as clavulanate and BAL 29880 were described. The combination of BAL30072 with BAL29880 or clavulanate resulted in susceptibility rates of more than $90 \%$ of all isolates tested in vitro. At $4 \mathrm{mg} / \mathrm{l}$, BAL30072 showed activity against all OprD-deficient Pseudomonas aeruginosa [23-25]. Also, most Acinetobacter baumannii strains expressing OXA or NMD carbapenemases were susceptible to BAL30072 [25].

In an in vitro study, the activity of BAL30072 and other standard antibiotics against meropenem-resistant $A$. baumannii was evaluated, and a MIC90 of $\geq 64$ was shown [23]. Burkholderia pseudomallei, the pathogen causing melioidosis, displays constitutive resistance toward a range of antimicrobials. Thus, treatment is difficult. In a study with its laboratory strains (1026b, 1710b) and several strains isolated in Thailand, more than $93 \%$ of the isolates were susceptible to BAL30072 with minimal inhibitory concentrations (MICs) in the range of $0.004-0.016 \mu \mathrm{g} / \mathrm{ml}$ [24].

Compared to ceftazidim, meropenem, and imipenem, BAL30072 showed markedly higher activity with a MIC90 of $0.016 \mu \mathrm{g} / \mathrm{ml}$. At the time of writing, BAL30072 is being investigated in phase I clinical studies [26, 27].

\section{Ceftolozane}

Ceftolozane combined with tazobactam is being investigated for complicated urinary tract infections (cUTIs), complicated intra-abdominal infections (cIAIs) and ventilator-associated pneumonia (VAP). It is a novel 
cephalosporin with a structure similar to ceftazidim. It exerts activity against Pseudomonas (including ceftazidimresistant $P$. aeruginosa) and also against bacteria-producing beta-lactamases such as TEM-1 (Temoneira: In 1965, this beta-lactamase was found in a patient called Temoneira) and SHV-1 (SHV = sulfhydryl variable) [28-30]. Ceftolozane shows no activity against bacteria-producing ESBL and carbapenemases. However, in combination with tazobactam, it is active against most of the bacteria-producing ESBL and against some anaerobes. The mean plasma halflife is $2.3 \mathrm{~h}$, and protein binding of ceftolozane is low at approximately $20 \%$. Two-phase II studies of ceftolozane with tazobactam in urinary tract infections and abdominal infections have been completed. One-phase III study has been terminated since a larger phase III study in VAP was planned in comparison with meropenem which has already started to enroll. The FDA approved ceftolozane/tazobactam to treat adults cIAI and cUTI in December 2014, and approval in Europe is expected for the end of 2015 [31, 32]. So far, the clinical and microbiological activity appears comparable to imipenem and ceftazidim: For $P$. aeruginosa, the MIC was $0.5 \mathrm{mg} / \mathrm{l}$ for ceftolozane, $1 \mathrm{mg} / \mathrm{l}$ for ceftazidim, and $0.5 \mathrm{mg} / \mathrm{l}$ for imipenem [33-35].

\section{Delafloxacin (fluoroquinolone)}

Quinolones are inhibitors of the bacterial DNA gyrase and topoisomerases (mostly topoisomerase IV). There are four generations of well-characterized quinolones which could be further developed, e.g., toward additional MRSA activity or better tissue penetration in neutral or acidic environments.

Delafloxacin is a promising investigational fluoroquinolone. In comparison with others, the substituent on position 7 of the quinoline ring system is not protonatable which results in a pKa shift. In contrast, other fluorquinolones are zwitterionic and therefore neutrally charged only at a physiological $\mathrm{pH}$. Neutral charge is required for membrane penetration. Delafloxacin also permeates membranes at lower $\mathrm{pH}$ such as found in inflamed tissue [36, 37]. In inflammatory tissue of soft tissue infections, abdominal infections, or urinary tract infections, $\mathrm{pH}$ levels are mildly acidic (about 5.5-6). Under these conditions, e.g., $90 \%$ of moxifloxacin is in a cationic state and therefore unable to permeate bacterial membranes. In contrast, delafloxacin is uncharged at this $\mathrm{pH}$ resulting in high cellular uptake [36]. Delafloxacin was already investigated in phase II studies for communityacquired pneumonia (CAP) and ABSSSI. The results were very promising so that phase III studies were initiated [38].

Omadacycline and eravacycline

Tetracyclines are bactericidal and inhibit protein synthesis by binding to the $30 \mathrm{~S}$ subunit of microbial ribosomes.
While omadacycline and eravacycline do not have advantages over tigecyclin in terms of their microbiological activity, both drugs are orally bioavailable and therefore interesting for further development [15].

Currently, omadacycline is being compared to linezolid in a phase III study in ABSSSI. It is administered parenterally in the initial phase. After clinical recovery, a switch to oral administration is possible. Omadacycline doses are $100 \mathrm{mg}$ for intravenous and $150 \mathrm{mg}$ for oral application [38].

Phase III studies of eravacycline are conducted with the antibiotic comparators levofloxacin or ertapenem in urinary tract infections and cIAI. Eravacyclin is administered parenterally every $12 \mathrm{~h}$ in cIAI.

\section{Solithromycin (ketolide)}

Solithromycin is a ketolide and derived from the macrolide erythromycin. The molecular mode of action is similar to telithromycin. It inhibits protein synthesis by binding ribosomal subunits. Microbiologically, the drug differs from macrolides due to additional MRSA activity and activity to resistant Streptococci.

Solithromycin is being investigated in clinical studies for CAP [39] and for gonococcal urethritis and compared with quinolones (levofloxacin and moxifloxacin) and the combination of ceftriaxone and azithromycin [38]. In vitro data and the existing clinical data suggest a lower risk of hepatotoxicity than for telithromycin [40].

\section{Novel beta-lactamase inhibitors}

The beta-lactamase inhibitors currently available for clinical application, i.e., sulbactam, clavulanate and tazobactam, inhibit beta-lactamases of the molecular class A reflecting the original TEM and SHV genes. Recently, other beta-lactamases are emerging which contribute to the increasing resistance especially of Gram-negative bacilli. Among those are oxacillinases [belonging to class D; OXA-1/10 (OXA = oxacillin hydrolyzing capabilities)], cephalosporinases [belonging to class C; AmpC (ampicillin class C BLI)], CMY-2 (cephamycin-BLI no. 2), FOX-1 (cefoxitin-BLI) and the metallo-beta-lactamases [belonging to class B; IMP-1 (imipenem-BLI), VIM-1/2 (Verona integron-encoded metallo-beta-lactamase), NDM-1 (New Delhi metallo-beta-lactamase), CphA (metallo-beta-lactamase from Aeromonas hydrophila), and Sfh-1 (betalactamase from Serratia fonticola)]. Novel beta-lactamase inhibitors (e.g., diazabicyclooctane-related substances) are able to also inhibit enzymes of these other groups to a various degree. They therefore contribute substantially to meet the increasing need for new drugs against ESBL or Klebsiella pneumonia carbapenemases (KPCs)-producing 
bacilli. Important representatives of these new beta-lactamase inhibitors are avibactam and MK-7655.

\section{Avibactam}

Avibactam belongs to the group of diazabicyclooctanes $[22,41]$. It inhibits nearly all class A and class C (AmpC) beta-lactamases. Due to its activity against various betalactamases, it is suitable for clinical testing in the setting of severe infections with multi-resistant Gram-negative bacteria. It was combined with either ceftazidime or ceftaroline. In vitro, ceftazidime/avibactam is active against multi-resistant enterobacteriaceae [e.g., bacilli with inducible or derepressed AmpC-beta-lactamases, class A betalactamases, carbapenemases belonging to class A (KPC) and class D (OXA-48)]. However, metallo-beta-lactamase producers were resistant toward avibactam [42].

The clinical efficacy and the safety of ceftazidime/avibactam are being tested in phase III clinical trials with different clinical indications. Among these are urosepsis, severe urinary tract infections, and intra-abdominal infections. In phase II clinical trials, it was shown that the efficacy of ceftazidime/avibactam was non-inferior to the carbapenem control group [22, 43].

In the two-phase III RECLAIM-1 and RECLAIM-2 trials, intravenous ceftazidime/avibactam plus metronidazole was compared to meropenem for cIAI. Ceftazidime/avibactam $(2000 \mathrm{mg} / 500 \mathrm{mg}$ ) was infused over $2 \mathrm{~h}$. Meropenem $(1000 \mathrm{mg}$ ) was given over $30 \mathrm{~min}$. The combination ceftazidime/avibactam/metronidazole met its primary end point of non-inferiority as shown by preliminary results [43].

\section{Mk-7655}

MK-7655 is a novel beta-lactamase inhibitor under clinical development. In vitro, it was tested against a KPC-2 carbapenemase-producing Klebsiella pneumoniae (isolate KP6339) and also against $P$. aeruginosa isolates lacking the OprD porin and overexpressing AmpC (isolates PA24226, PA24227, and PA24228). Synergism of MK-7655 in combination with imipenem was shown [44]. At the moment, a phase II study for severe urinary tract infections is being conducted. This study aims to proof that 125 or $250 \mathrm{mg}$ MK-7655 in combination with imipenem is non-inferior to imipenem only [38].

\section{RPX7009 and AAI101}

RPX7009 is a cyclic, boric acid-based beta-lactamase inhibitor active against serine-carbapenemases. It was given to 56 individuals in a dosage between 250 and $2000 \mathrm{mg}$ as a single dose or up to 7 days as 3-h infusion. The substance is mainly eliminated via the renal pathway and did not accumulate. Analysis of tolerability showed no significant difference to placebo. Severe adverse events did not occur [45].

AAI101 is a hybrid ionic beta-lactamase inhibitor with broad activity against ESBL. Efficacy was shown in combination with ceftriaxone, cefepime, or piperacillin in animal models and was compared to combinations with tazobactam. Clinical efficacy was defined as lethality of an intraperitoneal application of ESBL Gram-negative bacteria, and efficacy was significantly higher for all three combinations with AAI101 than for the control groups [46].

\section{Cadazolid, a novel substance for treating $C$. difficile}

Cadazolid is a novel antimicrobial agent with structural analogy to both oxazolidinones and fluoroquinolones. It has high and relatively selective efficacy against $C$. difficile. Its mechanism of action is inhibition of protein biosynthesis, which also leads to a highly effective suppression of toxin production. In addition, it strongly inhibits spore formation [47]. According to microbiological in vitro and animal models, one advantage of cadazolid is its relatively weak impact on the intestinal microbiota [48]. Also, there is no relevant intestinal absorption, leading to high intraluminal concentrations and a low systemic impact [49]. Cadazolid was tested in a multicentric, randomized, and double-blind phase II trial in patients with $C$. difficile-associated diarrhea (CDAD) using doses ranging from 250 to $1000 \mathrm{mg}$ per day over 10 days [50]. Standard dose oral vancomycin was used as comparator. A total of 84 patients with CDAD were included, and the study was completed in December of 2012. The efficacy of all dosages of cadazolid was similar or better compared to vancomycin with respect to all clinical end points, specifically cure rate and frequency of relapses. At the same time, the drug was well tolerated. Currently, a dosage of $250 \mathrm{mg}$ bid is being tested versus vancomycin $125 \mathrm{mg}$ qid in the large phase III IMPACT trial [51].

\section{Conclusion}

In conclusion, there are several promising compounds on their way through clinical development which will broaden the possibilities for treatment of MDR bacteria, especially against MRSA and Gram-negative ESBL bacteria. But the evolution of resistance mechanisms will not stop with the introduction of the new drugs. Therefore, the race must continue, and drugs with new mechanisms of action need to be investigated and tested for clinical use. Equally important, however, is the reduction in new infections and the counteraction of rising rates of antibiotic resistance with infection control mechanisms. 
Acknowledgments This work was supported by the "Deutsches Zentrum für Infektionsforschung" (DZIF), site Munich.

Conflict of interest J.R.B. has received honoraria for lectures from AbbVie, Astellas, Bayer, BMS, Boehringer Ingelheim, Gilead, Hexal, Janssen, MSD, Novartis and ViiV. R.D. has received payments for travel and conference support from Janssen, Gilead and Boehringer. U.S. has received payments for travel and conference support from Boehringer, Gilead, Janssen, Abbott and Pfizer/GSK/ViiV, lecture honoraria from Gilead, BMS and Janssen and Advisory Board/Speakers Bureau from Abbott, MSD, ViiV and Gilead. E.G. has not reported any conflict of interest.

Open Access This article is distributed under the terms of the Creative Commons Attribution License which permits any use, distribution, and reproduction in any medium, provided the original author(s) and the source are credited.

\section{References}

1. Dalhoff A. Resistance surveillance studies: a multifaceted problem - the fluoroquinolone example. Infection. 2012;40:239-62.

2. Lubbert $\mathrm{C}$, et al. Colonization of liver transplant recipients with KPC-producing Klebsiella pneumoniae is associated with high infection rates and excess mortality: a case-control analysis. Infection. 2014;42:309-16.

3. Zhanel GG, Schweizer F, Karlowsky JA. Oritavancin: mechanism of action. Clin Infect Dis. 2012;54:S214-9.

4. Rubino CM, et al. Oritavancin population pharmacokinetics in healthy subjects and patients with complicated skin and skin structure infections or bacteremia. Antimicrob Agents Chemother. 2009;53:4422-8.

5. Belley A, et al. Pharmacodynamics of a simulated single 1,200 -milligram dose of oritavancin in an in vitro pharmacokinetic/pharmacodynamic model of methicillin-resistant Staphylococcus aureus infection. Antimicrob Agents Chemother. 2013;57:205-11.

6. Dunbar LM, et al. Comparison of the efficacy and safety of oritavancin front-loaded dosing regimens to daily dosing: an analysis of the SIMPLIFI trial. Antimicrob Agents Chemother. 2011;55:3476-84.

7. Corey GR, Jiang H, Moeck G. Dalbavancin or oritavancin for skin infections. N Engl J Med. 2014;371:1162-3.

8. Seltzer E, et al. Once-weekly dalbavancin versus standard-of-care antimicrobial regimens for treatment of skin and soft-tissue infections. Clin Infect Dis. 2003;37:1298-303.

9. Jauregui LE, et al. Randomized, double-blind comparison of once-weekly dalbavancin versus twice-daily linezolid therapy for the treatment of complicated skin and skin structure infections. Clin Infect Dis. 2005;41:1407-15.

10. Raad I, et al. Efficacy and safety of weekly dalbavancin therapy for catheter-related bloodstream infection caused by gram-positive pathogens. Clin Infect Dis. 2005;40:374-80.

11. Nord CE, Rasmanis G, Wahlund E. Effect of dalbavancin on the normal intestinal microflora. J Antimicrob Chemother. 2006;58:627-31.

12. Dorr MB, et al. Human pharmacokinetics and rationale for onceweekly dosing of dalbavancin, a semi-synthetic glycopeptide. J Antimicrob Chemother. 2005;55:ii25-30.

13. Boucher HW, et al. Once-weekly dalbavancin versus daily conventional therapy for skin infection. N Engl J Med. 2014;370:2169-79.

14. Flanagan S, Passarell J, Lu Q. Tedizolid population pharmacokinetics, exposure-response, and target attainment. Antimicrob Agents Chemother. 2014;58:6462-70.
15. Liapikou A, et al. New antimicrobial approaches to gram positive respiratory infections. Pulm Pharmacol Ther. 2014 (Epub ahead of print).

16. Moran GJ, et al. Tedizolid for 6 days versus linezolid for 10 days for acute bacterial skin and skin-structure infections (ESTABLISH-2): a randomised, double-blind, phase 3, non-inferiority trial. Lancet Infect Dis. 2014;14:696-705.

17. Rybak JM, Marx K, Martin CA. Early experience with tedizolid: clinical efficacy, pharmacodynamics, and resistance. Pharmacotherapy. 2014;34:1198-208.

18. Flanagan S, et al. Single- and multiple-dose pharmacokinetics and absolute bioavailability of tedizolid. Pharmacotherapy. 2014;34:891-900.

19. Flanagan S, Minassian SL, Morris D. Pharmacokinetics of tedizolid in subjects with renal or hepatic impairment. Antimicrob Agents Chemother. 2014;58:6471-6.

20. Beringer $P$, et al. Absolute bioavailability and pharmacokinetics of linezolid in hospitalized patients given enteral feedings. Antimicrob Agents Chemother. 2005;49:3676-81.

21. Shorr AF, et al. Analysis of the phase 3 ESTABLISH trials: tedizolid versus linezolid in acute bacterial skin and skin structure infection. Antimicrob Agents Chemother. 2015;59:864-71.

22. Flamm RK, et al. Ceftazidime-avibactam and comparator agents tested against urinary tract isolates from a global surveillance program (2011). Diagn Microb Infect Dis. 2014;80:233-8.

23. Higgins PG, et al. In vitro activity of the siderophore monosulfactam BAL30072 against meropenem-non-susceptible Acinetobacter baumannii. J Antimicrob Chemother. 2012;67:1167-9.

24. Mima T, et al. In vitro activity of BAL30072 against Burkholderia pseudomallei. Int J Antimicrob Agents. 2011;38:157-9.

25. Mushtaq S, et al. Activity of BAL30072 alone or combined with beta-lactamase inhibitors or with meropenem against carbapenem-resistant Enterobacteriaceae and non-fermenters. J Antimicrob Chemother. 2013;68:1601-8.

26. OnVista. GNW-News: Basilea beginnt Phase-1-Kombinationsstudie mit ihrem gramnegativen Antibiotikum BAL30072 und Meropenem. 2014 05.10.2104. http://www.onvista.de/news/gnwnews-basilea-beginnt-phase-1-kombinationsstudie-mit-ihremgramnegativen-antibiotikum-bal30072-und-meropenem-1007603.

27. OnVista. Präsentation umfangreicher Daten zu Basileas AntiInfektiva Isavuconazol, Ceftobiprol und BAL30072 auf ICAAC. http://www.onvista.de/news/praesentation-umfangreicherdaten-zu-basileas-anti-infektiva-isavuconazol-ceftobiprol-undbal30072-auf-icaac-1127929.

28. Farrell DJ, et al. Antimicrobial activity of ceftolozane-tazobactam tested against Enterobacteriaceae and Pseudomonas aeruginosa with various resistance patterns isolated in U.S. Hospitals (2011-2012). Antimicrob Agents Chemother. 2013;57:6305-10.

29. Farrell DJ, et al. Ceftolozane/tazobactam activity tested against Gram-negative bacterial isolates from hospitalised patients with pneumonia in US and European medical centres (2012). Int J Antimicrob Agents. 2014:43:533-9.

30. Sader HS, et al. Antimicrobial activity of ceftolozane/tazobactam tested against Pseudomonas aeruginosa and Enterobacteriaceae with various resistance patterns isolated in European hospitals (2011-12). J Antimicrob Chemother. 2014;69:2713-22.

31. Personal communication of CUBIST Pharmaceuticals, December 17th 2014.

32. FDA. FDA approves new antibacterial drug Zerbaxa. 2014. http:// www.fda.gov/NewsEvents/Newsroom/PressAnnouncements/ ucm427534.htm.

33. Lucasti C, et al. Multicenter, double-blind, randomized, phase II trial to assess the safety and efficacy of ceftolozane-tazobactam plus metronidazole compared with meropenem in adult patients with complicated intra-abdominal infections. Antimicrob Agents Chemother. 2014;58:5350-7. 
34. Maseda E, et al. Ceftolozane/tazobactam (CXA 201) for the treatment of intra-abdominal infections. Expert Rev Anti Infect Ther. 2014;12:1-15.

35. Bretonniere $\mathrm{C}$, et al. In vivo efficacy of ceftolozane against Pseudomonas aeruginosa in a rabbit experimental model of pneumonia: comparison with ceftazidime, piperacillin/tazobactam and imipenem. Int J Antimicrob Agents. 2014;44:218-21.

36. Lemaire S, Tulkens PM, Van Bambeke F. Contrasting effects of acidic $\mathrm{pH}$ on the extracellular and intracellular activities of the anti-gram-positive fluoroquinolones moxifloxacin and delafloxacin against Staphylococcus aureus. Antimicrob Agents Chemother. 2011;55:649-58.

37. Siala W, et al. Antibiotic activity against biofilms from Staphylococcus aureus clinical isolates: factors determining the activity of the investigational fluoroquinolone delafloxacin in comparison with daptomycin and vancomycin. Antimicrob Agents Chemother. 2014;58:6385-97.

38. National_Institutes_of_Health_USA. Registry of publicly and privately supported clinical trials. 2014 05.10.2014. https://clinicaltrials.gov.

39. Oldach D, et al. Randomized, double-blind, multicenter phase 2 study comparing the efficacy and safety of oral solithromycin (CEM-101) to those of oral levofloxacin in the treatment of patients with community-acquired bacterial pneumonia. Antimicrob Agents Chemother. 2013;57:2526-34.

40. Van Bambeke F. Renaissance of antibiotics against difficult infections: focus on oritavancin and new ketolides and quinolones. Ann Med. 2014;46:512-29.

41. Sader HS, Flamm RK, Jones RN. Antimicrobial activity of ceftaroline-avibactam tested against clinical isolates collected from U.S. Medical Centers in 2010-2011. Antimicrob Agents Chemother. 2013;57:1982-8.

42. Livermore DM, et al. Activities of NXL104 combinations with ceftazidime and aztreonam against carbapenemase-producing Enterobacteriaceae. Antimicrob Agents Chemother. 2011;55:390-4.

43. Actavis. Actavis announces positive topline results from the phase III program of ceftazidime-avibactam in patients with complicated intra-abdominal infections (cIAI) 2014
05.10.2014. http://www.actavis.com/news/news/thomson-reuters/ actavis-announces-positive-topline-results-from-th.

44. Hirsch EB, et al. In vitro activity of MK-7655, a novel beta-lactamase inhibitor, in combination with imipenem against carbapenem-resistant Gram-negative bacteria. Antimicrob Agents Chemother. 2012;56:3753-7.

45. Griffith DC, et al. A phase 1 study of the safety, tolerability, and pharmacokinetics of the beta-lactamase inhibitor RPX7009 in health adult subjects, in 24th European congress of clinical microbiology an infectious diseases ECCMID 20142014: Barcelona.

46. Nordmann $P$, et al. Characterisation of beta-lactamase inhibition by AAI101, an extended-spectrum beta-lactamase inhibitor, in 24th European congress of clinical microbiology an infectious diseases ECCMID 20142014: Barcelona.

47. Locher $\mathrm{HH}$, et al. In vitro and in vivo antibacterial evaluation of cadazolid, a new antibiotic for treatment of Clostridium difficile infections. Antimicrob Agents Chemother. 2014;58:892-900.

48. Chilton $\mathrm{CH}$, et al. In vitro activity of cadazolid against clinically relevant Clostridium difficile isolates and in an in vitro gut model of $C$. difficile infection. $\mathrm{J}$ Antimicrob Chemother. 2014;69:697-705.

49. Baldoni D, et al. Cadazolid, a novel antibiotic with potent activity against Clostridium difficile: safety, tolerability and pharmacokinetics in healthy subjects following single and multiple oral doses. J Antimicrob Chemother. 2014;69:706-14.

50. Louie $\mathrm{T}$, et al. Multicentre, double-blind, randomised, phase 2 study evaluating the novel antibiotic, cadazolid, in subjects with Clostridium difficile-associated diarrhoea. Abstract LB 2956, 22nd European congress of clinical microbiology and infectious disease, Berlin, April 27-30, 2013.

51. Wall Street Journal. Actelions neuartiges Antibiotikum Cadazolid erhält Einstufung als Qualified Infectious Disease Product (QIDP-Status) zur Behandlung von Clostridium-difficile-assoziierter Diarrhoe durch die FDA 2014 05.10.2104. http://www. wallstreet-online.de/nachricht/6606569-actelions-neuartiges-antibiotikum-cadazolid-einstufung-qualified-infectious-disease-product-qidp-status-behandlung-clostridium-difficile-assoziierterdiarrhoe-fda. 